Faculty of Applied Mathematics

University of Twente

University for Technical and Social Sciences
P.O. Box 217

7500 AE Enschede

The Netherlands

Phone: +31-53-4893400

Fax: $+31-53-4893114$

Email: memo@math.utwente.nl

Memorandum No. 1433

Isomorphisms and traversability of directed path graphs

H.J. BROERsma AND X. LI ${ }^{1}$

FEBRUary 1998

ISSN 0169-2690

\footnotetext{
${ }^{1}$ Department of Applied Mathematics, Northwestern Polytechnical University, Xi'an, Shaanxi 710072, P.R. China
} 


\title{
Isomorphisms and Traversability of Directed Path Graphs
}

\author{
Hajo Broersma ${ }^{1}$ \\ XUELIANG LI ${ }^{2 *}$ \\ ${ }^{1}$ Faculty of Applied Mathematics \\ University of Twente \\ P.O. Box 217, 7500 AE Enschede \\ The Netherlands \\ ${ }^{2}$ Department of Applied Mathematics \\ Northwestern Polytechnical University \\ Xi'an, Shaanxi 710072 \\ P.R. China
}

September 29, 1998

\begin{abstract}
The concept of a line digraph is generalized to that of a directed path graph. The directed path graph $\vec{P}_{k}(D)$ of a digraph $D$ is obtained by representing the directed paths on $k$ vertices of $D$ by vertices. Two vertices are joined by an arc whenever the corresponding directed paths in $D$ form a directed path on $k+1$ vertices or form a directed cycle on $k$ vertices in $D$. In this introductory paper several properties of $\overrightarrow{P_{3}}(D)$ are studied, in particular with respect to isomorphism and traversability. In our main results, we characterize all digraphs $D$ with $\vec{P}_{3}(D) \cong D$, we show that $\vec{P}_{3}\left(D_{1}\right) \cong \vec{P}_{3}\left(D_{2}\right)$ "almost always" implies $D_{1} \cong D_{2}$, and we characterize all digraphs with Eulerian or Hamiltonian $\overrightarrow{P_{3}}$-graphs.
\end{abstract}

Keywords: directed path graph, line digraph, isomorphism, traversability.

AMS Subject Classifications (1991): 05C75, 05C45, 05C05.

* Part of this research was carried out while the second author was visiting the Faculty of Applied Mathematics, University of Twente, supported by the Dutch Organization for Scientific Research (NWO) and the NSFC. 


\section{Introduction}

We refer to [2] for any undefined terminology.

In [3] path graphs were introduced as a generalization of line graphs of (undirected) graphs. In the next section we shall introduce an analogous concept for directed graphs. But first we recall some basic definitions and notation concerning directed graphs.

We define a directed graph or digraph $D$ to be a pair $(V(D), A(D))$, where $V(D)$ is a finite non-empty set of elements called vertices, and $A(D)$ is a (finite) set of distinct ordered pairs of distinct elements of $V(D)$ called arcs. For convenience we shall denote the arc $(v, w)$ (where $v, w \in V(D))$ by $v w$. If $a=v w$ is an $\operatorname{arc}$ of $D$, then we say that $v$ and $w$ are adjacent, and that $a$ is an out-arc of $v$ and an in-arc of $w$; we call $w$ an out-neighbour of $v$ and $v$ an in-neighbour of $w$. The in-degree $d^{-}(v)$ of $v$ is the number of in-arcs of $v$; the out-degree $d^{+}(v)$ of $v$ is the number of out-arcs of $v$; $v$ is a source or sink if $d^{-}(v)=0$ or $d^{+}(v)=0$, respectively. The underlying graph $U(D)$ of a digraph $D$ is the graph (or multigraph) obtained from $D$ by replacing each arc by an (undirected) edge joining the same pair of vertices. A digraph $D$ is called strongly connected if, for each pair of vertices $v$ and $w$, there is a directed path in $D$ from $v$ to $w$, and connected if there is a path from $v$ to $w$ in $U(D)$. A directed subgraph of $D$ corresponding to a path of $U(D)$ is called a semipath of $D$. We denote by $\vec{P}_{k}$ a directed path on $k$ vertices $(k \geq 1)$, i.e. a semipath on $k$ vertices with one source and one sink, in which all arcs are oriented from source to sink. A directed cycle $\vec{C}_{k}(k \geq 2)$ consists of a $\vec{P}_{k}$ with source $v$ and sink $w$ together with the arc $w v$. Two $\operatorname{arcs} a, b \in A(D)$ are said to be adjacent if $\{a, b\}=\{v w, w z\}$ for some vertices $v, w, z \in V(D)$; to stress the head-to-tail adjacency, we

say that $a$ hits $b$ if $a=v w$ and $b=w z$. We call two adjacent arcs $a, b \in A(D)$ a $\vec{P}_{3}$-pair or a $\overrightarrow{C_{2}}$-pair if they form a $\overrightarrow{P_{3}}$ or a $\overrightarrow{C_{2}}$ in $D$, respectively. If $\{a, b\} \subseteq A(D)$ is a $\vec{P}_{3}$-pair and $a$ hits $b$, then we denote the $\overrightarrow{P_{3}}$ formed by $a$ and $b$ simply by $a b$.

\section{Directed path graphs}

Let $k$ be a positive integer, and let $D$ be a digraph containing at least one $\vec{P}_{k}$. Denote by $\vec{\Pi}_{k}(D)$ the set of all $\vec{P}_{k}$ 's of $D$. Then the $\vec{P}_{k^{-}}$graph of $D$, denoted by $\vec{P}_{k}(D)$, is the digraph with vertex set $\vec{\Pi}_{k}(D) ; p q$ is an arc of $\vec{P}_{k}(D)$ if and only if there is a $\vec{P}_{k+1}$ or $\vec{C}_{k} v_{1} v_{2} \ldots v_{k+1}$ in $D$ (with $v_{1}=v_{k+1}$ in the case of a $\vec{C}_{k}$ ) such that $p=v_{1} v_{2} \ldots v_{k}$ and $q=v_{2} \ldots v_{k} v_{k+1}$. Note that $\vec{P}_{1}(D)=D$ and $\overrightarrow{P_{2}}(D)=\vec{L}(D)$, the line digraph of $D$, as it was introduced in [4].

For a nice survey of results on line graphs and line digraphs we refer to [5]. In the sequel we shall restrict ourselves to $\overrightarrow{P_{3}}$-graphs. In Section 3 we give some elementary results on $\vec{P}_{3^{-}}$ graphs, in Section 4 we discuss isomorphisms of $\vec{P}_{3}$-graphs, and in Section 5 we consider the traversability of $\vec{P}_{3}$-graphs. 


\section{$3 \quad$ Elementary results}

\subsection{Vertices, arcs and degrees}

Let $D$ be a digraph containing at least one $\overrightarrow{P_{3}}$ and let $G=\overrightarrow{P_{3}}(D)$. To express the number of vertices, the number of arcs, and the degrees of the vertices of $G$ in terms of $D$, we first introduce some additional terminology.

For a vertex $v \in V(D)$, we set

$$
\bar{A}_{v}=\{u \in V(D) \mid\{u v, v u\} \subseteq A(D)\}
$$

and we define

$$
\bar{A}(D)=\{u v \in A(D) \mid v u \in A(D)\} .
$$

Now the number of $\overrightarrow{P_{3}}$ 's in $D$ with middle vertex $v$ is equal to

$$
\left(d^{-}(v)-\left|\bar{A}_{v}\right|\right) d^{+}(v)+\left|\bar{A}_{v}\right|\left(d^{+}(v)-1\right)=d^{-}(v) d^{+}(v)-\left|\bar{A}_{v}\right| .
$$

Hence

$$
|V(G)|=\sum_{v \in V(D)}\left(d^{-}(v) d^{+}(v)-\left|\bar{A}_{v}\right|\right)=\sum_{v \in V(D)} d^{-}(v) d^{+}(v)-|\bar{A}(D)|
$$

The number of arcs of $G$ can be counted by summing up, for each arc $a$ of $D$, the number of $\overrightarrow{P_{3}}$, 's of $D$ "joined" together by having the arc $a$ "in common", as follows: each $\operatorname{arc} u v \in A(D) \backslash \bar{A}(D)$ joins $d^{-}(u) d^{+}(v) \overrightarrow{P_{3}}$ 's, while each arc $u v \in \bar{A}(D)$ joins $\left(d^{-}(u)-1\right)\left(d^{+}(v)-1\right) \overrightarrow{P_{3}}$ 's. Hence

$$
|A(G)|=\sum_{u v \in A(D)}\left(d^{-}(u) d^{+}(v)\right)+|\bar{A}(D)|-\sum_{u v \in \bar{A}(D)}\left(d^{-}(u)+d^{+}(v)\right) .
$$

The in-degree and out-degree of a vertex in $G$ corresponding to a $\overrightarrow{P_{3}} u v w$ in $D$ are

$$
\begin{aligned}
& d^{-}(u)-\left|\{u\} \cap \bar{A}_{v}\right| \quad \text { and } \\
& d^{+}(w)-\left|\{w\} \cap \bar{A}_{v}\right|,
\end{aligned}
$$

respectively.

\subsection{Cycle structure}

By considering the possible adjacency structures of $\overrightarrow{P_{3}}$ 's in a digraph $D$, one easily obtains the

following result on (short) cycles in $\vec{P}_{3}(D)$. We omit the proof and remark that similar results can be deduced for longer cycles. Recall that $U(D)$ denotes the underlying (undirected) graph of $D$. 


\section{Theorem 1}

Let $D$ be a digraph. Then

(i) $\overrightarrow{P_{3}}(D)$ contains no $\overrightarrow{C_{2}}$;

(ii) Each $C_{3}$ in $U\left(\overrightarrow{P_{3}}(D)\right)$ is a $\overrightarrow{C_{3}}$ in $\overrightarrow{P_{3}}(D)$;

(iii) Each $C_{4}$ in $U\left(\overrightarrow{P_{3}}(D)\right)$ is induced (has no chords) and is a $\vec{C}_{4}$ or is oriented with alternating arc directions in $\overrightarrow{P_{3}}(D)$;

(iv) $N_{o} C_{k}(k \geq 5)$ of $U\left(\overrightarrow{P_{3}}(D)\right)$ is both induced and oriented with alternating arc directions in $\overrightarrow{P_{3}}(D)$.

\subsection{Splitting vertices}

Let $D$ be a digraph and $v \in V(D)$ a source with out-arcs $v u_{1}, \ldots, v u_{k}$. Suppose $D^{\prime}$ is obtained from $D$ by replacing $v$ by two (or more) new vertices $v_{1}, v_{2}$ and splitting the out-arcs $v u_{1}, \ldots, v u_{k}$ into two (or more) disjoint (non-empty) sets $v_{1} u_{1}, \ldots, v_{1} u_{k_{1}}, v_{2} u_{k_{1}+1}, \ldots, v_{2} u_{k}$. Then it is clear that $\overrightarrow{P_{3}}\left(D^{\prime}\right) \cong \overrightarrow{P_{3}}(D)$. A similar splitting preserving the $\vec{P}_{3}$-structure can be applied to sinks. Of course the reverse operation of combining sources or sinks is also preserving the $\overrightarrow{P_{3}}$-structure, as long as sources or sinks do not have common out-neighbours or in-neighbours, respectively.

Splitting an arbitrary vertex $v$ of $D$ into two new vertices $v_{1}, v_{2}$ and dividing the in-arcs and out-arcs at $v$ among $v_{1}$ and $v_{2}$, we obtain a digraph $D^{\prime}$ with the property that $\vec{P}_{3}\left(D^{\prime}\right)$ is an induced subgraph of $\vec{P}_{3}(D)$. We leave the details to the reader.

\section{Isomorphisms of $\overrightarrow{P_{3}}$-graphs}

In this section we consider two questions:

(1) For which digraphs $D$ is $\overrightarrow{P_{3}}(D) \cong D$ ?

(2) For which digraphs $D_{1}$ and $D_{2}$ does $\overrightarrow{P_{3}}\left(D_{1}\right) \cong \overrightarrow{P_{3}}\left(D_{2}\right)$ imply $D_{1} \cong D_{2}$ ?

We refer to [5] for results related to similar questions concerning line (di)graphs, and to [3] for analogous results on $P_{3}$-graphs of (undirected) graphs.

In this section we shall characterize all digraphs for which $\overrightarrow{P_{3}}(D) \cong D$, and we shall see that $\overrightarrow{P_{3}}\left(D_{1}\right) \cong \overrightarrow{P_{3}}\left(D_{2}\right)$ "almost always" implies $D_{1} \cong D_{2}$. Before we present the results we introduce some additional terminology.

Let $D$ be a digraph. A direct tree $T$ of $D$ is an out-tree of $D$ if $V(T)=V(D)$ and precisely one vertex of $T$ has in-degree zero (the root of $T$ ), while all other vertices of $T$ have in-degree one. An in-tree of $D$ is defined analogously with respect to out-degrees. Note that 
any strongly-connected digraph contains an in-tree and an out-tree, and no sources or sinks. We first give a short proof of the following result.

\section{Theorem 2}

Let $D$ be a connected digraph without sources or sinks. If $D$ has an in-tree or an out-tree, then $\vec{P}_{3}(D) \cong D$ if and only if $D \cong \vec{C}_{n}$ for some $n \geq 3$. Hence, if $D$ is strongly connected, then $\overrightarrow{P_{3}}(D) \cong D$ if and only if $D \cong \overrightarrow{C_{n}}$ for some $n \geq 3$.

Proof If $D \cong \vec{C}_{n}$ for some $n \geq 3$, then clearly $\overrightarrow{P_{3}}(D) \cong \overrightarrow{C_{n}} \cong D$.

For the converse, assume without loss of generality that $D$ has an out-tree $T$ with root $v$. Let $t$ denote the number of vertices with out-degree zero in $T$. Denote $V(D)=\left\{v, v_{1}, \ldots, v_{n-1}\right\}$, where $v_{1}, \ldots, v_{t}$ are the vertices with out-degree zero in $T$. Note that $\vec{P}_{3}(D)$ does not contain $\overrightarrow{C_{2}}$. Since $v$ has at least one in-arc in $D$, and each of $v_{1}, \ldots, v_{t}$ has at least one out-arc in $D$, we know that $\overrightarrow{P_{3}}(D)$ has at least

$$
d_{T}^{+}(v)+t+d_{T}^{+}\left(v_{t+1}\right)+\ldots+d_{T}^{+}\left(v_{n-1}\right)
$$

vertices. From $\overrightarrow{P_{3}}(D) \cong D$ we obtain

$$
n=|V(D)|=\left|V\left(\overrightarrow{P_{3}}(D)\right)\right| \geq d_{T}^{+}(v)+t+d_{T}^{+}\left(v_{t+1}\right)+\ldots+d_{T}^{+}\left(v_{n-1}\right),
$$

hence

$$
d_{T}^{+}(v)+d_{T}^{+}\left(v_{t+1}\right)+\ldots+d_{T}^{+}\left(v_{n-1}\right) \leq n-t .
$$

On the other hand, since $T$ is an out-tree, we obtain

$$
d_{T}^{+}(v)+\left(d_{T}^{+}\left(v_{t+1}\right)+1\right)+\ldots+\left(d_{T}^{+}\left(v_{n-1}\right)+1\right)+t=2(n-1),
$$

hence

$$
d_{T}^{+}(v)+d_{T}^{+}\left(v_{t+1}\right)+\ldots+d_{T}^{+}\left(v_{n-1}\right)=n-1 .
$$

Combining (1) and (2), we get that $t \leq 1$, implying that $t=1$, and that $T=\vec{P}_{n}$. Similar arguments show that any in-tree of $D$ is a $\vec{P}_{n}$. This is only possible if $D \cong \vec{C}_{n}$.

Let $D$ be a digraph obtained from a $\vec{P}_{m_{1}}\left(m_{1} \geq 2\right)$ and a $\vec{C}_{m_{2}}\left(m_{2} \geq 3\right)$ by identifying the first or last vertex of $\vec{P}_{m_{1}}$ with one vertex of $\vec{C}_{m_{2}}$. Then one easily checks that $\overrightarrow{P_{3}}(D) \cong D$, that $D$ contains either precisely one source or precisely one sink, and that $D$ contains an in-tree or an out-tree. This class of graphs can be extended by taking a directed cycle and attaching a collection of disjoint out-trees (or in-trees) to the vertices of the cycle.

In the sequel we characterize all connected digraphs satisfying $\vec{P}_{3}(D) \cong D$.

First we present a useful relationship between $\overrightarrow{P_{3}}$-graphs and iterated line digraphs. Given a digraph $D$, we denote by $\operatorname{Asym}(D)$ the graph obtained from $D$ by deleting all $\overrightarrow{C_{2}}$ 's, i.e. by deleting all $\overrightarrow{C_{2}}$-pairs $\{u v, v u\} \subseteq A(D)$. 


\section{Theorem 3}

For any digraph $D$ containing at least one $\overrightarrow{P_{3}}, \overrightarrow{P_{3}}(D) \cong \vec{L}(A \operatorname{sym}(\vec{L}(D)))$.

Proof Let $D$ be a digraph containing at least one $\vec{P}_{3}$. Then $\vec{P}_{3}(D)$ and $\vec{L}(A \operatorname{sym}(\vec{L}(D)))$ exist, and $v \in V(\vec{L}(A \operatorname{sym}(\vec{L}(D))))$ if and only if $v \in A(A \operatorname{sym}(\vec{L}(D)))$. This is equivalent to saying that $v=x y$ for some $x, y \in V(A \operatorname{sym}(\vec{L}(D)))$, or, equivalently, for some $x, y \in V(\vec{L}(D))$ such that $\{x, y\}$ is not a $\vec{C}_{2}$-pair of $D$. It is clear that this is equivalent to saying that $v$ is a $\overrightarrow{P_{3}}$ of $D$, hence $v \in V\left(\vec{P}_{3}(D)\right)$.

Moreover, $u v$ is an arc of $\overrightarrow{L(} \operatorname{Asym}(\vec{L}(D)))$ if and only if $u v$ corresponds to a $\overrightarrow{P_{3}}$ in $\operatorname{Asym}(\vec{L}(D))$, and hence in $\vec{L}(D)$. It is again clear that this is equivalent to saying that $u$ and $v$ correspond to two $\overrightarrow{P_{3}}$ 's in $D$ forming a $\overrightarrow{P_{4}}$ or $\overrightarrow{C_{3}}$ in $D$, or, equivalently, that $u v$ is an arc of $\overrightarrow{P_{3}}(D)$.

\section{Corollary 4}

For any digraph $D$ containing at least one $\overrightarrow{P_{3}}$ and no $\overrightarrow{C_{2}}, \overrightarrow{P_{3}}(D) \cong \vec{L}(\vec{L}(D))=\overrightarrow{L^{2}}(D)$.

Proof This follows immediately from Theorem 3 and the observation that $D$ contains a $\vec{C}_{2}$ if and only if $\vec{L}(D)$ contains a $\vec{C}_{2}$.

\section{Corollary 5}

For any digraph $D$ containing at least one $\vec{P}_{3}$,

$\lim \sup _{n \rightarrow \infty}\left|V\left(\overrightarrow{P_{3}^{n}}(D)\right)\right|<\infty$ if and only if $\lim _{n \rightarrow \infty}\left|V\left(\overrightarrow{L^{2} n-1}(\operatorname{Asym}(\vec{L}(D)))\right)\right|<\infty$.

Proof This follows from the fact that $\vec{P}_{3}^{2}(D)=\vec{L}(\operatorname{Asym}(\vec{L}(\vec{L}(\operatorname{Asym}(\vec{L}(D))))))=$ $\overrightarrow{L^{3}}(\operatorname{Asym}(\vec{L}(D)))$, hence $\vec{P}_{3}^{n}(D)=\overrightarrow{L^{2} n-1}(\operatorname{Asym}(\vec{L}(D)))$.

\section{Corollary 6}

For any digraph $D$ containing at least one $\vec{P}_{3}, \vec{P}_{3}(D)$ is strongly connected if and only if $\operatorname{Asym}(\vec{L}(D))$ is strongly connected.

Proof This is an immediate consequence of Theorem 3 and [5, Theorem 7.4(i)]: $\vec{L}(D)$ is strongly connected if and only if $D$ is strongly connected.

In particular, if $\operatorname{Asym}(D)$ is strongly connected, then $\operatorname{Asym}(\vec{L}(D))$ is strongly connected and so is $\overrightarrow{P_{3}}(D)$, but for the following digraph $D, \operatorname{Asym}(D)$ is disconnected, while $\operatorname{Asym}(\vec{L}(D))$ is strongly connected.

The digraph $D$ consists of two vertex-disjoint $\vec{C}_{3}$ 's and two additional arcs $u v$ and $v u$ between two vertices $u$ and $v$ of different $\vec{C}_{3}$ 's. It is easy to find infinitely many examples of the same type. Another infinite class of examples can be obtained from the class of (undirected) trees (with at least two edges) by replacing all edges of the trees by distinct $\overrightarrow{C_{2}}$ 's; for such a resulting graph $D, A(A \operatorname{sym}(D))=\varnothing$, while $A \operatorname{sym}(\vec{L}(D))$ contains a Hamilton cycle, e.g. a directed cycle through all vertices (See also Section 5$)$. 


\subsection{Characterizing all connected digraphs $D$ with $\overrightarrow{P_{3}}(D) \cong D$}

In this subsection we assume $D$ is a connected digraph satisfying $\vec{P}_{3}(D) \cong D$. Then by Theorem $1(\mathrm{i}), D$ contains no $\overrightarrow{C_{2}}$, hence by Corollary $4, D \cong \overrightarrow{P_{3}}(D) \cong \overrightarrow{L^{2}}(D)$, hence $D \cong$ $\overrightarrow{P_{3}^{n}}(D) \cong \vec{L}^{2 n}(D)$ for any integer $n \geq 1$. By [5, Theorem 7.4(i)], if $D$ is strongly connected, then $\vec{L}(D)$ is strongly connected, hence $\vec{P}_{3}^{n}(D)$ is strongly connected for all $n \geq 1$; then the counting arguments from Section 3.1 imply $\left|V\left(\vec{P}_{3}^{n}(D)\right)\right| \geq\left|V\left(\vec{P}_{3}^{n-1}(D)\right)\right| \geq \ldots \geq|V(D)|$, with equality only if $D$ is a directed cycle. Since $|V(D)|$ is finite this shows that all strongly connected components of $D$ are directed cycles. By [5, Theorem 9.1(i)], $\left|A\left(\overrightarrow{L^{n}}(D)\right)\right|=\varnothing$ for some $n$ if and only if $D$ has no directed cycles, and by [5, Theorem 9.1(ii)], $\left|V\left(\overrightarrow{L^{n}}(D)\right)\right|$ gets arbitrarily large if and only if $D$ has two directed cycles joined by a directed path (possibly of length 0). Moreover, by [5, Theorem 7.2(iii)], if no two directed cycles of $D$ are joined by a directed path, then for all sufficiently large values of $n$, each connected component of $\overrightarrow{L^{n}}(D)$ has at most one directed cycle. These results together imply that $D$ has precisely one directed cycle $C$. Following [5, pp. 298-299] we define the basic configuration of $D$ to be the subdigraph formed by the union of all directed paths of $D$ directed to and from vertices of $C$. As remarked there, the line digraph of $D$ and each of its iterates have a single directed cycle (all of the same length), and for sufficiently large $n$, the basic configuration of $\overrightarrow{L^{n}}(D)$ consists of a directed cycle and an out-tree and in-tree (disjoint except for roots) at each vertex of the directed cycle. Hence $D$ is of this form. It is not difficult to check one cannot have an out-tree and in-tree attached at the same vertex of $C$, since this creates an isolated vertex in $\vec{P}_{3}(D)$ or a subdigraph attached to a vertex of the directed cycle that is neither an out-tree nor an in-tree. If $C$ has length 3 , one can have arbitrary out- or in-trees attached to each vertex of $C$; if $C$ has length 4 or more, the situation is more complicated: certain combinations of outand in-trees are possible, others are not. To characterize all possible combinations, we have to introduce some more definitions from [5].

Given the digraph $D$ consisting of a directed cycle $C=v_{0} v_{1} \ldots v_{k-1} v_{0}$ together with an outtree $A_{i}$ or an in-tree $B_{i}$ at each vertex $v_{i}(0 \leq i \leq k-1)$, the cyclic (modulo $k$ ) sequences $\left\{A_{i}\right\}$ and $\left\{B_{i}\right\}$ are called the out-tree and in-tree sequences. As mentioned in [5], the line digraph of $D$ has the same sequences with the in-trees advanced one vertex of the cycle relative to the out-trees. The out-tree index of $D$ is then defined as the minimum positive integer $r$ for which $A_{i+r} \cong A_{i}$ for all $i$; the in-tree index is defined similarly. If, for some positive integers $n$ and $k$, we have $\vec{L}^{n}+k(D) \cong \overrightarrow{L^{n}}(D)$, then we call $D \vec{L}$-periodic, and the smallest value of $k$ for which this holds its $\vec{L}$-period. Theorem 9.2 of [5] relates the $\vec{L}$-period of $D$ to its out-tree and in-tree indices: Let $D$ be an $\vec{L}$-periodic digraph with a single directed cycle. Then the $\vec{L}$-period of $D$ is the greatest common divisor of its out-tree and in-tree indices.

From the above discussion it is not difficult to conclude that for a connected digraph $D$, $\overrightarrow{P_{3}}(D) \cong D$ if and only if $D$ is its basic configuration with at most one out- or in-tree attached

to the vertices of the directed cycle $C$, and the $\vec{L}$-period of $D$ is 1 or 2 . It is harder to give a 
precise description of $D$. We already saw that, if $C$ has length 3, any combination of out- and in-trees is possible; if $C \cong \vec{C}_{2 k}(k \geq 2)$, then any combination with copies of the same out-tree (in-tree) attached to $v_{0}, v_{2}, v_{4}, \ldots$ and arbitrary in-trees (out-trees) attached to $v_{1}, v_{3}, \ldots$ is possible. Of course also any combination of out-trees (or in-trees) attached to the vertices of $C$ is possible.

\subsection{Periodic $\overrightarrow{P_{3}}$-graphs}

We present a result on $\vec{P}_{3}$-graphs analogous to [5, Theorem 9.1] and [5, Corollary 7.2]. We omit the proofs, since they are easy consequences of $\vec{P}_{3}^{n}(D)=\vec{L}^{2 n-1}(\operatorname{Asym}(\vec{L}(D)))$, and [5, Theorem 9.1] and [5, Corollary 7.2], as well as the fact that directed cycles in $\vec{L}(D)$ correspond to directed closed walks in $D$, and directed paths in $\overrightarrow{L(} D)$ to directed walks in $D$. Here a digraph $D$ is called $\overrightarrow{P_{3}}$-periodic if ${\overrightarrow{P_{3}^{n}}}^{n+k}(D) \cong{\overrightarrow{P_{3}^{n}}}^{n}(D)$ for some positive integers $k$ and $n$.

\section{Theorem 7}

Let $D$ be a digraph. Then:

(i) $A\left(\overrightarrow{P_{3}^{n}}(D)\right)=\varnothing$ for some $n$ if and only if $D$ has no directed cycles except for $\vec{C}_{2}$ 's.

(ii) $\left|V\left(\overrightarrow{P_{3}^{n}}(D)\right)\right|$ gets arbitrarily large for sufficiently large $n$ if and only if $D$ has two directed cycles of length at least 3 joined by a directed path (possibly of length 0 ).

(iii) $D$ is $\overrightarrow{P_{3}}$-periodic if and only if $D$ has directed cycles of length at least 3 , no two of which are joined by a directed path (possibly of length 0 ).

(iv) If $D$ is strongly connected and $\overrightarrow{P_{3}^{n}}(D) \cong D$ for some $n \geq 1$, then $\overrightarrow{P_{3}}(D) \cong D$ and $D$ is a directed cycle.

\subsection{Which digraphs have isomorphic $\vec{P}_{3}$-graphs?}

Before we turn to Question 2 we introduce some additional terminology concerning isomorphisms.

Let $D$ and $D^{\prime}$ be two digraphs. An isomorphism of $D$ onto $D^{\prime}$ is a bijection $f: V(D) \rightarrow$ $V\left(D^{\prime}\right)$ such that $u v \in A(D)$ if and only if $f(u) f(v) \in A\left(D^{\prime}\right)$. An arc-isomorphism of $D$ onto $D^{\prime}$ is a bijection $f: A(D) \rightarrow A\left(D^{\prime}\right)$ such that $a \in A(D)$ hits $b \in A(D)$ if and only if $f(a) \in A\left(D^{\prime}\right)$

hits $f(b) \in A\left(D^{\prime}\right)$. Hence an arc-isomorphism of $D$ onto $D^{\prime}$ is an isomorphism of $\vec{L}(D)$ onto $\vec{L}\left(D^{\prime}\right)$. A $\overrightarrow{P_{3}}$-isomorphism of $D$ onto $D^{\prime}$ is an isomorphism of $\overrightarrow{P_{3}}(D)$ onto $\overrightarrow{P_{3}}\left(D^{\prime}\right)$.

Question 2 can be rephrased as follows.

$\left(2^{\prime}\right)$ Which $\vec{P}_{3}$-isomorphisms of $D$ onto $D^{\prime}$ are induced by isomorphisms of $D$ onto $D^{\prime}$ ?

The related question for arc-isomorphisms was answered in [4]. 


\section{Theorem 8}

Let $D$ and $D^{\prime}$ be two digraphs without sources or sinks. Then every arc-isomorphism of $D$ onto $D^{\prime}$ is induced by an isomorphism of $D$ onto $D^{\prime}$, hence $\vec{L}(D) \cong \vec{L}\left(D^{\prime}\right)$ if and only if $D \cong D^{\prime}$.

We can prove a similar result on $\overrightarrow{P_{3}}$-isomorphisms if we make a "weak" additional assumption concerning the digraphs $D$ and $D^{\prime}$. This additional assumption is reasonable and is the natural counterpart of the assumption in Theorem 8 that $D$ and $D^{\prime}$ contain no sources or sinks.

\section{Theorem 9}

Let $D$ and $D^{\prime}$ be two connected digraphs without sources or sinks. If for each arc $a=u v \in$ $A(D) \cup A\left(D^{\prime}\right)$ these exist arcs $b=x u$ and $c=v y$ in the same digraph with $x \neq v$ and $y \neq u$, then every $\vec{P}_{3}$-isomorphism of $D$ onto $D^{\prime}$ is induced by an arc-isomorphism of $D$ onto $D^{\prime}$.

Proof Let $f$ denote a $\vec{P}_{3}$-isomorphism of $D$ onto $D^{\prime}$, where $D$ and $D^{\prime}$ satisfy the conditions of the theorem. For any arc $x \in A(D)$, there exist two arcs $y, z \in A(D)$ such that $y x$ and $x z$ correspond to two $\overrightarrow{P_{3}}$ 's of $D$. Since $f$ is a $\overrightarrow{P_{3}}$-isomorphism, for some $\vec{P}_{3}$-pairs $\{a, b\},\{c, d\} \subseteq$ $A\left(D^{\prime}\right), f(y x)=a b$ and $f(x z)=c d$. But this implies $b=c$, since adjacencies of $\overrightarrow{P_{3}}$ 's are preserved by $f$. Fixing $z$ we see that $b$ is independent of $y$, and fixing $y$ we see that $c$ is independent of $z$, so $b=c$ depends only on $x$. Denoting $b=c$ by $f^{*}(x)$ for each $x$, we get a function $f^{*}: A(D) \rightarrow A\left(D^{\prime}\right)$ such that $f(u v)=f^{*}(u) f^{*}(v)$ for each $u v$ corresponding to a $\overrightarrow{P_{3}}$ of $D$. By similar reasoning there is a function $f_{*}$ so that $f^{-1}(a b)=f_{*}(a) f_{*}(b)$ for each $a b$ corresponding to a $\overrightarrow{P_{3}}$ of $D^{\prime}$. Clearly $f_{*} \cdot f^{*}$ and $f^{*} \cdot f_{*}$ are identity functions, so $f^{*}$ and $f_{*}$ are inverse bijections. The function $f^{*}$ induces $f$. We claim that $f^{*}$ is an arc-isomorphism from $D$ to $D^{\prime}$. From above, both $f^{*}$ and $f_{*}$ preserve arcs $u v$ of the line digraph corresponding to a $\overrightarrow{P_{3}}$. Now we must show that $f^{*}$ and $f_{*}$ preserve arcs $x y$ of the line digraph corresponding to a $\vec{C}_{2}$. By symmetry, it suffices to prove this for $f^{*}$. Assume $\{x, y\}$ is a $\vec{C}_{2}$-pair in $D$. If $D$ is a digraph obtained from a cycle $C_{n}$ by replacing each edge $u v$ by two arcs $u v$ and $v u$, then one easily checks that $D \cong D^{\prime}$, unless $D^{\prime}$ consists of two disjoint $\vec{C}_{n}$ 's, contradicting the connectivity of $D^{\prime}$. In the other case, there exist $\operatorname{arcs} x_{1}, x_{2}, \ldots, x_{k}, y_{1}, y_{2}, \ldots, y_{k}$ in $D$ such that $\left\{x_{1}, y_{1}\right\},\left\{y_{k}, x_{k}\right\}$, and $\left\{x_{i}, x_{i+1}\right\},\left\{y_{i+1}, y_{i}\right\}(i=1, \ldots, k-1)$ are $\vec{P}_{3}$-pairs in $D$, while $\left\{x_{i}, y_{i}\right\}(i=2, \ldots, k-1)$ are $\vec{C}_{2}$-pairs in $D$, and $\{x, y\}=\left\{x_{i}, y_{i}\right\}$ for some $i \in\{2, \ldots, k-1\}$. We complete the proof by showing that, for each $i \in\{2, \ldots, k-1\},\left\{f^{*}\left(x_{i}\right), f^{*}\left(y_{i}\right)\right\}$ is a $\overrightarrow{C_{2}}$-pair in $D^{\prime}$, in particular $\left\{f^{*}(x), f^{*}(y)\right\}$. Assume, to the contrary, that $i$ is the smallest index in $\{2, \ldots, k-1\}$ such that $\left\{f^{*}\left(x_{i}\right), f^{*}\left(y_{i}\right)\right\}$ is not a $\overrightarrow{C_{2}}$-pair. Suppose first that $f^{*}\left(x_{i}\right) f^{*}\left(y_{i}\right)$ (or $\left.f^{*}\left(y_{i}\right) f^{*}\left(x_{i}\right)\right)$ is a $\overrightarrow{P_{3}}$ in $D^{\prime}$. Then, since $f_{*}$ preserves $\overrightarrow{P_{3}}$-pairs, $x_{i} y_{i}$ is a $\overrightarrow{P_{3}}$ in $D$, a contradiction. Hence $f^{*}\left(x_{i}\right)$ and $f^{*}\left(y_{i}\right)$ are nonadjacent arcs in $D^{\prime}$. Considering the $\overrightarrow{P_{3}}$ 's $f^{*}\left(x_{i-1}\right) f^{*}\left(x_{i}\right)$ and $f^{*}\left(y_{i}\right) f^{*}\left(y_{i-1}\right)$ in $D^{\prime}$, it is clear that $\left\{f^{*}\left(x_{i-1}\right), f^{*}\left(y_{i-1}\right)\right\}$ is not a $\vec{C}_{2}$-pair in $D^{\prime}$. The choice of $i$ implies that $i=2$. Hence $\left\{f^{*}\left(x_{2}\right), f^{*}\left(y_{2}\right)\right\}$ is not a $\vec{C}_{2}$-pair (nor a $\vec{P}_{3}$-pair) in $D^{\prime}$. Now, considering the $\overrightarrow{P_{3}}$ 's $f^{*}\left(x_{1}\right) f^{*}\left(x_{2}\right), f^{*}\left(x_{1}\right) f^{*}\left(y_{1}\right)$, and $f^{*}\left(y_{2}\right) f^{*}\left(y_{1}\right)$ in $D^{\prime}$, we easily obtain a contradiction. 
From the above proof we already note that we cannot omit the connectivity condition in Theorem 9 .

To illustrate the necessity of the condition on the sources and sinks we can use the splitting technique from Section 3.3. As a small example consider the next pair of nonisomorphic digraphs with isomorphic $\overrightarrow{P_{3}}$-graphs. The first digraph consists of a $\overrightarrow{C_{3}}$, one additional vertex $v$, and arcs from $v$ to two vertices of the $\vec{C}_{3}$; the second one of a $\vec{C}_{3}$, two additional vertices $v_{1}, v_{2}$, and $\operatorname{arcs} v_{1} u$ and $v_{2} w$ to two vertices $u$ and $w$ of the $\overrightarrow{C_{3}}$.

With respect to the necessity of the condition on the arcs, consider the following pair of nonisomorphic digraphs with isomorphic $\vec{P}_{3}$-graphs. The first digraph is a $\vec{C}_{4}$; the second one is obtained from a $\overrightarrow{C_{4}}$ by replacing two vertex-disjoint arcs by $\overrightarrow{C_{2}}$ 's. In general, if we add to $D$ any arc not contained in a $\overrightarrow{P_{3}}$ to get $D^{\prime}$, then $\overrightarrow{P_{3}}(D) \cong \vec{P}_{3}\left(D^{\prime}\right)$. In particular we can add an arc from a source to a sink, or, if $u v$ is an arc where $d^{+}(u)=d^{-}(v)=1$, we can add the arc $v u$.

Combining Theorems 8 and 9 it is clear we have the following consequences for digraphs $D$ and $D^{\prime}$ satisfying the conditions in the hypothesis of Theorem 9 .

Corollary 10

$\overrightarrow{P_{3}}(D) \cong \vec{P}_{3}\left(D^{\prime}\right)$ if and only if $D \cong D^{\prime}$.

\section{Corollary 11}

$\operatorname{Aut}(D) \cong \operatorname{Aut}(\vec{L}(D)) \cong \operatorname{Aut}\left(\overrightarrow{P_{3}}(D)\right)$.

\section{Remark}

Recently, in [1] the equation $P_{3}(G) \cong P_{3}\left(G^{\prime}\right)$ for undirected graphs has been solved completely, building on earlier work in [3] and [6].

\section{$5 \quad$ Traversability of $\overrightarrow{P_{3}}$-graphs}

In this section we consider (directed) Euler tours and (directed) Hamilton cycles in $\overrightarrow{P_{3}}$-graphs.

For line digraphs of strongly connected digraphs, the following result ([5, Theorem 10.1]) characterizes the traversability.

\section{Theorem 12}

Let $D$ be a strongly connected digraph. Then

(i) $\vec{L}(D)$ is Eulerian if and only if $d^{-}(v)=d^{+}(w)$ for each arc $v w$ in $D$;

(ii) $\vec{L}(D)$ is Hamiltonian if and only if $D$ is Eulerian.

Combining Theorems 3 and 12 we immediately obtain the following characterization of Eulerian and Hamiltonian $\overrightarrow{P_{3}}$-graphs of digraphs $D$ in terms of properties of $A \operatorname{sym}(\vec{L}(D))$. 


\section{Corollary 13}

Let $D$ be a digraph such that $\operatorname{Asym}(\vec{L}(D))$ is strongly connected. Then

(i) $\overrightarrow{P_{3}}(D)$ is Eulerian if and only if $d^{-}(v)=d^{+}(w)$ for each arc vw in $\operatorname{Asym}(\vec{L}(D))$;

(ii) $\vec{P}_{3}(D)$ is Hamiltonian if and only if $\operatorname{Asym}(\vec{L}(D))$ is Eulerian.

The properties of $\operatorname{Asym}(\vec{L}(D))$ in Corollary 13 can be translated into properties of $D$ as follows.

For an arc $x y \in A(D)$, let

$$
\begin{aligned}
& d^{-}(x y)=d^{-}(x)-|\{y x\} \cap \bar{A}(D)| \quad \text { and } \\
& d^{+}(x y)=d^{+}(y)-|\{y x\} \cap \bar{A}(D)| .
\end{aligned}
$$

Then $d^{-}(v)=d^{+}(w)$ for each arc $v w$ in $\operatorname{Asym}(\vec{L}(D))$ if and only if $d^{-}(a b)=d^{+}(b c)$ for each $\overrightarrow{P_{3}} a b c$ in $D$.

We say that a Euler tour $T$ of $D$ is a $\overrightarrow{C_{2}}$-tour if the arcs of each $\vec{C}_{2}$ of $D$ are successive arcs in $T$. Then $\operatorname{Asym}(\vec{L}(D))$ is Eulerian if and only if $\overrightarrow{L(D)}$ has a $\overrightarrow{C_{2}}$-tour and $\vec{L}(D) \neq \overrightarrow{C_{2}}$. Furthermore, $\operatorname{Asym}(\vec{L}(D))$ is a vertex-disjoint union of Eulerian digraphs if $\vec{L}(D)$ is Eulerian (and $¥ \overrightarrow{C_{2}}$ ). Hence we obtain the following result.

\section{Theorem 14}

Let $D$ be a digraph such that $\operatorname{Asym}(\vec{L}(D))$ is strongly connected. Then

(i) $\overrightarrow{P_{3}}(D)$ is Eulerian if and only if $d^{-}(a b)=d^{+}(b c)$ for each $\overrightarrow{P_{3}}$ abc in $D$;

(ii) $\vec{P}_{3}(D)$ is Hamiltonian if and only if $\vec{L}(D)$ has a $\vec{C}_{2}$-tour;

(iii) $\overrightarrow{P_{3}}(D)$ contains a 2-factor if and only if $\vec{L}(D)$ is Eulerian, or, equivalently if $d^{-}(v)=$ $d^{+}(w)$ for each arc $v w$ in $D$;

(iv) $\vec{P}_{3}(D)$ is Hamiltonian if $d^{-}(v)=d^{+}(w)$ for each arc $v w$ in $D$, and $D$ contains no $\vec{C}_{2}$.

\section{References}

[1] R.E.L. Aldred, M.N. Ellingham, R.L. Hemminger and P. Jipsen, $P_{3}$-isomorphisms for graphs. Journal of Graph Theory 26 (1997) 35-51.

[2] J.A. Bondy and U.S.R. Murty, Graph Theory with Applications. (MacMillan/Elsevier, London/New York, 1976).

[3] H.J. Broersma and C. Hoede, Path graphs. Journal of Graph Theory 13 (1989) 427-444.

[4] F. Harary and R.Z. Norman, Some properties of line digraphs. Rend. Circ. Mat. Palermo (2) 9 (1960) 161-168. 
[5] R.L. Hemminger and L.W. Beineke, Line graphs and line digraphs, in: Selected Topics in Graph Theory (eds. L.W. Beineke and R.J. Wilson). (Academic Press, London, New York, San Francisco, 1978).

[6] X. Li, Isomorphisms of P $P_{3}$-graphs. Journal of Graph Theory 21 (1996) 81-85. 